\title{
The extremal function for $K_{9}^{=}$minors
}

\author{
Martin Rolek* \\ Department of Mathematics \\ College of William \& Mary \\ Williamsburg, VA 23185
}

March 26, 2018

\begin{abstract}
We prove the extremal function for $K_{9}^{=}$minors, where $K_{9}^{=}$denotes the complete graph $K_{9}$ with two edges removed. In particular, we show that any graph with $n$ vertices and at least $6 n-20$ edges either contains a $K_{9}^{\bar{y}}$ minor or is isomorphic to a graph obtained from disjoint copies of $K_{8}$ and $K_{2,2,2,2,2}$ by identifying cliques of size 5 . We utilize computer assistance to prove one of our lemmas.
\end{abstract}

Keywords: extremal function, graph minor

\section{Introduction}

All graphs considered are simple and finite. We use $V(G),|G|, E(G), e(G), \delta(G), \Delta(G)$, and $\bar{G}$ to denote the vertex set, number of vertices, edge set, number of edges, minimum degree, maximum degree, and complement of a graph $G$, respectively. Given $S, T \subseteq V(G)$,

\footnotetext{
*E-mail address: msrolek@wm.edu.
} 
we denote by $e(S, T)$ the number of edges of $G$ with one end in $S$ and one end in $T$. We denote by $G[S]$ the subgraph of $G$ induced by $S$, and by $G-S$ the subgraph $G[V(G) \backslash S]$ of $G$. If $S=\{x\}$, we simply write $G-x$ in the latter case. For $u v \in E(\bar{G})$, we denote by $G+u v$ the graph obtained from $G$ by adding an edge joining $u$ and $v$. The union (resp. intersection) of two graphs $G$ and $H$, denoted $G \cup H$ (resp. $G \cap H$ ), is the graph with vertex set $V(G) \cup V(H)$ (resp. $V(G) \cap V(H))$ and edge set $E(G) \cup E(H)(\operatorname{resp} . \quad E(G) \cap E(H))$. The join of two graphs $G$ and $H$, denoted $G \vee H$, is the graph with vertex set $V(G) \cup V(H)$ and edge set $E(G) \cup E(H) \cup\{u v: u \in V(G), v \in V(H)\}$. If $G$ contains $H$ as a minor, we denote this by $G \geq H$. $G / x y$ denotes the graph obtained from $G$ by contracting the edge $x y$. A vertex $x$ is complete to a set $S$ if $x$ is adjacent to every vertex of $S$. We use $d_{G}(x)$ to denote the degree of a vertex $x$ in the graph $G$. Given a subgraph $H$ of $G$, we define $N(H)$ to be the set of vertices of $V(G) \backslash V(H)$ with a neighbor in $V(H)$.

Given a graph property $\mathcal{P}$, the extremal function for $\mathcal{P}$ determines the maximum number of edges a graph on $n$ vertices may have while not satisfying property $\mathcal{P}$. Extremal graph theory began when Turán [22] determined the extremal function for $K_{t}$ subgraphs. He further characterized all such graphs attaining this maximum number of edges, the wellknown Turán graphs. Dirac [2] was the first to consider the natural extension to $K_{t}$ minors. When considering $K_{t}$ minors instead of $K_{t}$ subgraphs, the problem is much more difficult to solve, and the extremal function is known only for $t \leq 9$. Dirac [2] showed for $t \leq 5$, and Mader [11] for $t \in\{6,7\}$, that any graph on $n \geq t$ vertices with at least $(t-2) n-\left(\begin{array}{c}t-1 \\ 2\end{array}\right)+1$ edges has a $K_{t}$ minor. The case $t=6$ was also independently shown by Györi [5].

For $t \geq 8$, there exist families of graphs with $(t-2) n-\left(\begin{array}{c}t-1 \\ 2\end{array}\right)+1$ edges, but which do not contain a $K_{t}$ minor. To describe these families, we define an $\left(H_{1}, H_{2}, k\right)$-cockade recursively as follows. Any graph isomorphic to either $H_{1}$ or $H_{2}$ is an $\left(H_{1}, H_{2}, k\right)$-cockade. Now given two $\left(H_{1}, H_{2}, k\right)$-cockades $G_{1}$ and $G_{2}$, we let $G$ be the graph obtained from $G_{1}$ and $G_{2}$ by identifying a clique of size $k$ in $G_{1}$ with a clique of size $k$ in $G_{2}$. Then $G$ is an $\left(H_{1}, H_{2}, k\right)$ - 
cockade, and every $\left(H_{1}, H_{2}, k\right)$-cockade can be constructed in this fashion. If $H_{1}$ is isomorphic to $H_{2}$, we simply write $\left(H_{1}, k\right)$-cockade. Jørgensen [10] showed that any graph on $n \geq 8$ vertices with at least $6 n-20$ edges either has a $K_{8}$ minor or is a $\left(K_{2,2,2,2,2}, 5\right)$-cockade, and Song and Thomas [19] showed any graph on $n \geq 9$ vertices with at least $7 n-27$ edges either has a $K_{9}$ minor or is a $\left(K_{1,2,2,2,2,2}, 6\right)$-cockade, or is isomorphic to $K_{2,2,2,3,3}$, settling the cases $t=8$ and $t=9$, respectively. The extremal function for $K_{t}$ minors remains open for $t \geq 10$. Note that in a certain sense there is only one minimal counterexample for the case $t=8$, namely $K_{2,2,2,2,2}$, and two for the case $t=9$, namely $K_{1,2,2,2,2,2}$ and $K_{2,2,2,3,3}$. As pointed out by Song [18], there are at least eight minimal counterexamples for the case $t=10$, and Thomas and Zhu (see [20]) have conjectured that there are no further minimal counterexamples.

As a simplification, the extremal function for $K_{t}^{-}$minors has been investigated, where $K_{t}^{-}$is the complete graph on $t$ vertices with one edge removed. For $t \in\{5,6\}$, Dirac [2] showed that any graph on $n \geq t$ vertices with at least $\frac{1}{2}((2 t-5) n-(t-3)(t-1))$ edges either has a $K_{t}^{-}$minor or is a $\left(K_{t-1}, t-3\right)$-cockade. For larger values of $t$, there is more than one minimal counterexample. Jakobsen [8,9] showed any graph on $n \geq 7$ vertices with at least $\frac{1}{2}(9 n-24)$ edges either has a $K_{7}^{-}$minor or is a $\left(K_{6}, K_{2,2,2,2}, 4\right)$-cockade, and Song [17] showed any graph on $n \geq 8$ vertices with at least $\frac{1}{2}(11 n-35)$ edges either has a $K_{8}^{-}$minor or is a $\left(K_{7}, K_{1,2,2,2,2}, 5\right)$-cockade. The extremal function for $K_{t}^{-}$minors remains open for $t \geq 9$.

In this paper, we will consider $K_{t}^{=}$minors, where $K_{t}^{=}$denotes the complete graph on $t$ vertices with two edges removed. Note that there are two nonisomorphic graphs $K_{t}^{=}$, depending on whether the removed edges share a common end or not. Let $\mathcal{K}_{t}^{=}$denote the family consisting of the two nonisomorphic graphs $K_{t}^{=}$. Throughout this paper, we will use the following conventions. We say a graph $G$ has no $K_{t}^{=}$minor if $G$ does not contain $K$ as a minor for any $K \in \mathcal{K}_{t}^{=}$, and we say that $G$ has a $K_{t}^{=}$minor if $G$ contains $K$ as a minor for some $K \in \mathcal{K}_{t}^{=}$. Dirac [2] proved the following for $t \in\{5,6\}$, and Jakobsen $[7,8]$ proved the 
cases $t \in\{7,8\}$.

Theorem 1.1 (Dirac [2], Jakobsen [7, 8]) For $t \in\{5, \ldots, 8\}$, if $G$ is a graph with $|G| \geq t-1$ and at least $(t-3) n-\frac{1}{2}(t-1)(t-4)$ edges, then either $G \geq K_{t}^{=}$or $G$ is a $\left(K_{t-1}, t-4\right)$-cockade.

Our main result is to extend Theorem 1.1 to the case $t=9$ as follows.

Theorem 1.2 If $G$ is a graph with $|G| \geq 8$ and at least $6|G|-20$ edges, then either $G \geq K_{9}^{=}$ or $G$ is a $\left(K_{8}, K_{2,2,2,2,2}, 5\right)$-cockade.

Note that for this case there are now two minimal counterexamples to consider. In Section 2 we prove several results necessary for the proof of Theorem 1.2, which we present in Section 3.

Our primary motivation for studying the extremal functions for $K_{t}, K_{t}^{-}$, and $K_{t}^{=}$minors is their integral use in proving results related to Hadwiger's conjecture [6], which claims that every graph with no $K_{t}$ minor is $(t-1)$-colorable. Hadwiger's conjecture is easily true for $t \leq 3$. The case $t=4$ was shown by both Hadwiger [6] and Dirac [3], and a short proof was given much later by Woodall [24]. For $t=5$, Wagner [23] showed that Hadwiger's conjecture is equivalent to the Four Color Theorem, and for $t=6$, Robertson, Seymour, and Thomas [12] showed the same. The conjecture remains open for $t \geq 7$, although there are some partial results as follows. Albar and Gonçalves [1] showed for $t \in\{7,8\}$, and the present author and Song [15] for $t=9$, that every graph with no $K_{t}$ minor is $(2 t-6)$ colorable. An alternate proof for the cases $t \in\{7,8\}$ is also provided in [15]. By noticing that known minimal counterexamples to the extremal function for $K_{t}$ minors for $t \geq 7$ are all $(t-1)$-colorable, the present author and Song [15] proved the first general result on coloring graphs with no $K_{t}$ minor for all $t \geq 6$, provided a suitable conjecture holds as follows.

Conjecture 1.3 (Rolek and Song [15]) For every $t \geq 1$, every graph on $n$ vertices with at least $(t-2) n-\left(\begin{array}{c}t-1 \\ 2\end{array}\right)+1$ edges either has a $K_{t}$ minor or is $(t-1)$-colorable. 
Theorem 1.4 (Rolek and Song [15]) For $t \geq 6$, if Conjecture 1.3 is true, then every graph with no $K_{t}$ minor is $(2 t-6)$-colorable.

The chromatic number of graphs without $K_{t}^{-}$minors and $K_{t}^{=}$minors has also been investigated. Jakobsen [8,9] showed for $t=7$, and the present author and Song [15] for $t=8$, that if $G$ has no $K_{t}^{-}$minor, then $G$ is $(2 t-7)$-colorable, and if $G$ has no $K_{t}^{=}$minor, then $G$ is $(2 t-8)$-colorable. Most recently, the present author [13] has used Theorem 1.2 to show the following.

Theorem 1.5 (Rolek [13]) If $G$ has no $K_{9}^{=}$minor, then $G$ is 10-colorable.

\section{Preliminaries}

We begin this section with four results on $\left(K_{8}, K_{2,2,2,2,2}, 5\right)$-cockades which will be useful early in Section 3.

Lemma 2.1 Let $G$ be a $\left(K_{8}, K_{2,2,2,2,2}, 5\right)$-cockade, and let $x$ and $y$ be nonadjacent vertices in $G$. Then $G+x y \geq K_{9}^{=}$.

Proof. We proceed by induction on $|G|$. It is easy to see that the statement holds if $G$ is isomorphic to $K_{2,2,2,2,2}$. Hence we may assume that $G$ is obtained from $H_{1}$ and $H_{2}$ by identifying a $K_{5}$, where both $H_{1}$ and $H_{2}$ are $\left(K_{8}, K_{2,2,2,2,2}, 5\right)$-cockades. If both $x, y \in V\left(H_{i}\right)$ for some $i \in\{1,2\}$, then $G \geq K_{9}^{=}$by induction. Thus we may assume that $x \in V\left(H_{1}\right) \backslash V\left(H_{2}\right)$ and $y \in V\left(H_{2}\right) \backslash V\left(H_{1}\right)$. If there exists $z \in V\left(H_{1} \cap H_{2}\right)$ such that $x z \notin E(G)$, then by contracting the component of $H_{2}-V\left(H_{1} \cap H_{2}\right)$ containing $y$ onto $z$ and deleting all other components of $H_{2}-V\left(H_{1} \cap H_{2}\right)$, we see that $G \geq H_{1}+x z$, and the resulting graph contains a $K_{9}^{=}$minor by induction. Hence we may assume that $x$ is complete to $V\left(H_{1} \cap H_{2}\right)$, and similarly that $y$ is complete to $V\left(H_{1} \cap H_{2}\right)$. Since $G\left[\left(V\left(H_{1} \cap H_{2}\right)\right) \cup\{x\}\right]$ is isomorphic to 
$K_{6}$, it follows that there is a $K_{8}$-subgraph $H^{\prime}$ of $H_{1}$ such that $\left(V\left(H_{1} \cap H_{2}\right)\right) \cup\{x\} \subseteq V\left(H^{\prime}\right)$. Then $G\left[V\left(H^{\prime}\right) \cup\{y\}\right]$ is a $K_{9}^{=}$-subgraph in $G+x y$.

From Lemma 2.1 we get the following.

Lemma 2.2 If $G^{\prime}$ is a $\left(K_{8}, K_{2,2,2,2,2}, 5\right)$-cockade, and $G$ is the graph obtained from $G^{\prime}$ by adding a new vertex joined to at least six vertices of $G^{\prime}$, then $G \geq K_{9}^{=}$.

Proof. Let $G$ and $G^{\prime}$ be as in the statement, and say $v \in V(G) \backslash V\left(G^{\prime}\right)$. If there exist $x, y \in N_{G}(v)$ such that $x y \notin E(G)$, then $G \geq G^{\prime}+x y \geq K_{9}^{=}$by Lemma 2.1. Hence $N_{G}(v)$ is complete, and so there exists a $K_{8}$-subgraph $H$ of $G^{\prime}$ such that $N_{G}(v) \subseteq V(H)$. But then $G[V(H) \cup\{v\}]$ is isomorphic to $K_{9}^{=}$.

Lemma 2.3 Let $G$ be a graph with $\delta(G) \geq 7$. Let $x y \in E(G)$ such that $x$ and $y$ have at least 5 common neighbors. If $G / x y$ is a $\left(K_{8}, K_{2,2,2,2,2}, 5\right)$-cockade, then $G \geq K_{9}^{=}$.

Proof. We proceed by induction on $|G|$. The statement is easy to verify if $G / x y$ is isomorphic to $K_{8}$. Assume $G / x y$ is isomorphic to $K_{2,2,2,2,2}$. Say $V(G / x y)=\left\{v_{1}, w_{1}, \ldots, v_{5}, w_{5}\right\}$, where $v_{i} w_{i} \notin E(G / x y)$ for $i \in\{1, \ldots, 5\}$, and $w_{5}$ is the vertex obtained by contracting the edge $x y$ of $G$. We may assume by Pigeonhole that $v_{1}, w_{1}, v_{2}, v_{3}$ are common neighbors of $x$ and $y$ in $G$. If $w_{2}$ is also a common neighbor of $x$ and $y$, then we may assume by symmetry that $x w_{3}, y w_{4} \in E(G)$ since $\delta(G) \geq 7$ and each of $w_{3}, w_{4}$ must be adjacent to at least one of $x, y$. Now by contracting the edges $v_{1} v_{5}$ and $w_{3} w_{4}$, and noting that $v_{4}$ also must be adjacent to at least one of $x, y$, we see that $G \geq K_{9}^{=}$. Hence we may assume $w_{2}$ is not a common neighbor of $x$ and $y$, and by symmetry neither is $w_{3}$. Then $v_{4}$, say, is a common neighbor of $x$ and $y$. Now since $\delta(G) \geq 7$ and each of $w_{2}, w_{3}, w_{4}$ must be adjacent to at least one of $x, y$, we may assume that $x w_{2}, y w_{3} \in E(G)$. By contracting the edges $v_{1} v_{5}$ and $w_{2} w_{3}$, we again see $G \geq K_{9}^{=}$. 
Therefore we may assume that $G / x y$ is a $\left(K_{8}, K_{2,2,2,2,2}, 5\right)$-cockade obtained from $H_{1}$ and $H_{2}$ by identifying a $K_{5}$, where both $H_{1}$ and $H_{2}$ are $\left(K_{8}, K_{2,2,2,2,2}, 5\right)$-cockades. Say $v \in$ $V(G / x y)$ is the vertex resulting from contracting the edge $x y$ of $G$. Let $S=V\left(H_{1} \cap H_{2}\right)$. Let $H_{i}^{\prime}$ be the subgraph of $G$ induced by $\left(V\left(H_{i}\right) \backslash\{v\}\right) \cup\{x, y\}$ for $i \in\{1,2\}$. If $v \in V\left(H_{1}\right) \backslash V\left(H_{2}\right)$, then every common neighbor of $x$ and $y$ in $G$ belongs to $V\left(H_{1}^{\prime}\right)$. Further, every vertex of $S$ has at least 7 neighbors in $V\left(H_{1}^{\prime}\right)$. Hence $G \geq K_{9}^{=}$by induction applied to $H_{1}^{\prime}$. Thus $v \notin V\left(H_{1}\right) \backslash V\left(H_{2}\right)$ and similarly, $v \notin V\left(H_{2}\right) \backslash V\left(H_{1}\right)$. Therefore $v \in S$. Let $Z$ denote the set of common neighbors of $x$ and $y$ in $G$. We may assume that $\left|Z \cap V\left(H_{1}\right)\right| \geq\left|Z \cap V\left(H_{2}\right)\right|$, and in particular that $Z \cap\left(V\left(H_{1}\right) \backslash V\left(H_{2}\right)\right) \neq \emptyset$ since $|Z| \geq 5$ and $|S \backslash\{v\}|=4$. We may also assume that $d_{H_{1}^{\prime}}(x) \geq d_{H_{1}^{\prime}}(y)$. Let $Z^{\prime}$ be a set of neighbors of $y$ in $H_{2}^{\prime}-x$ chosen maximal subject to $\left|Z^{\prime}\right| \leq 4$ and then further such that $\left|Z \cap Z^{\prime}\right|$ is maximum. Say $\left|Z^{\prime}\right|=r$. Since $G / x y$ is a $\left(K_{8}, K_{2,2,2,2,2}, 5\right)$-cockade, it is 5 -connected. Thus there exist $r$ disjoint paths $P_{1}, \ldots, P_{r}$ in $G / x y-v$ with one end in $Z^{\prime}$ and the other end in $S \backslash\{v\}$. Then each path $P_{i}$ is also a path in $G$. Let $H^{*}$ be the graph obtained from $G$ by contracting each path $P_{i}$ onto its end in $S$ and deleting all vertices of $V\left(H_{2}\right) \backslash V\left(H_{1} \cup P_{1} \cup \cdots \cup P_{r}\right)$. Then by the choice of $Z^{\prime}, x$ and $y$ have at least 5 common neighbors in $H^{*}$. Also, $H^{*} \geq H_{1}$. If $d_{H^{*}}(y) \geq 7$, then $\delta\left(H^{*}\right) \geq 7$, so $G \geq H^{*} \geq K_{9}^{=}$by induction applied to $H^{*}$. Otherwise, $d_{H^{*}}(y)=6, y$ has only one neighbor in $V\left(H^{*}\right) \backslash S$, say $w \in Z$, and every vertex of $S \backslash\{v\}$ is a common neighbor of $x$ and $y$. Since $H_{1}$ is a cockade and $H_{1}[S]$ is isomorphic to $K_{5}$, some subgraph $K$ of $H_{1}$ contains $S$, where $K$ is either isomorphic to $K_{8}$ or $K_{2,2,2,2,2}$. Then there exists a path $Q$ in $H_{1}$ with one end $w$ and the other end in $V(K)$ such that $Q$ avoids $S$, and $Q$ has no internal vertices in $V(K)$ (possibly $Q$ consists only of the vertex $w$ ). By contracting $Q$ onto its end in $V(K)$, we may assume that $y$ has one neighbor in $V(K) \backslash S$. Let $K^{*}$ be the subgraph of $H^{*}$ induced by $V(K) \cup\{x, y\} \backslash\{v\}$. Note that every vertex of $V(K)$ is adjacent to $x$, except the nonneighbor of $v$ in $K$ if $K$ is isomorphic to $K_{2,2,2,2,2}$, because each such vertex is adjacent to at least one of $x$ or $y$, and every neighbor of $y$ in $V(K)$ is a common neighbor of $x$ and 
$y$. Hence, $K^{*}-y$ is isomorphic to $K$, that is $K^{*}-y$ is isomorphic to $K_{8}$ or $K_{2,2,2,2,2}$ and in particular is a $\left(K_{8}, K_{2,2,2,2,2}, 5\right)$-cockade. Since $y$ has six neighbors in $V\left(K^{*}\right)$, it follows from Lemma 2.2 that $G \geq K^{*} \geq K_{9}^{=}$.

It is easy to verify the following, so the details are omitted.

Lemma 2.4 If $G$ is a $\left(K_{8}, K_{2,2,2,2,2}, 5\right)$-cockade, then $e(G)=6|G|-20$.

Given a graph $G$ and a set $T=\left\{v_{1}, \ldots, v_{4}\right\} \subseteq V(G)$, we say that $G$ has a $K_{4}$ minor rooted at $T$ if, for $i \in\{1, \ldots, 4\}, G$ has disjoint subsets $T_{i} \subseteq V(G)$ such that $G\left[T_{i}\right]$ is connected, $T_{i} \cap T=\left\{v_{i}\right\}$, and there exist some vertices $u_{i} \in T_{i}$ and $u_{j} \in T_{j}$ such that $u_{i} u_{j} \in E(G)$ for all $j \in\{1, \ldots, 4\}$ with $j \neq i$. The concept of rooted $K_{4}$ minors is an extension of earlier work by Seymour [16] and Thomassen [21] on 2-linked graphs. Robertson, Seymour, and Thomas utilized rooted $K_{4}$ minors in their proof of Hadwiger's conjecture for graphs with no $K_{6}$ minor [12], and the next result is a simplified restatement of (2.6) from that same paper. For a complete characterization of graphs with rooted $K_{4}$ minors, see Fabila-Monroy and Wood [4].

Theorem 2.5 (Robertson, Seymour, and Thomas [12]) If $G$ is 4-connected and $T \subseteq V(G)$ with $|T|=4$, then either $G$ has a $K_{4}$-minor rooted at $T$, or $G$ is planar with $e(G) \leq 3|G|-7$.

Lemma 2.6 Let $G$ be a graph with $|G| \in\{7, \ldots, 11\}$ and $\delta(G) \geq 6$. If $G$ is not 4-connected, then $G$ contains $K_{5}$ as a subgraph. If $G$ is not 5-connected, then $G$ contains $K_{5}^{-}$as a subgraph.

Proof. The statement is clearly true if $G$ is a complete graph, so we may assume that $|G| \in\{8, \ldots, 11\}$. Let $S$ be a minimum separating set in $G$, and let $G_{1}, G_{2}$ be proper subgraphs of $G$ such that $G_{1} \cup G_{2}=G$ and $G_{1} \cap G_{2}=G[S]$. Since $\delta(G) \geq 6$, it follows that $\left|G_{i}\right| \geq 7$ for $i \in\{1,2\}$. Since $|G|=\left|G_{1}\right|+\left|G_{2}\right|-|S| \geq 14-|S|$, we have $|S| \geq 5$ for $|G| \in\{8,9\}$, and $|S| \geq 4$ for $|G|=10$. Assume first that $G$ is not 4-connected. Then 
$|G|=11$ and $|S|=3$, so $\left|G_{1}\right|=7$. Any vertex $u \in V\left(G_{1}\right) \backslash S$ is complete to $V\left(G_{1}\right) \backslash\{u\}$. Since $\left|G_{1}-S\right|=4$, the subgraph of $G$ induced by $\left(V\left(G_{1}\right) \backslash S\right) \cup\{v\}$ for any $v \in S$ is isomorphic to $K_{5}$. Now assume that $G$ is 4 -connected, but not 5-connected. Then $|G| \in\{10,11\}$. At least one of $G_{1}, G_{2}$, say $G_{1}$, must satisfy $\left|G_{1}\right|=7$. Any vertex $u \in V\left(G_{1}\right) \backslash S$ is complete to $V\left(G_{1}\right) \backslash\{u\}$. Since $\left|G_{1}-S\right| \geq 3$, the subgraph of $G$ induced by $\left(V\left(G_{1}\right) \backslash S\right) \cup\left\{v_{1}, v_{2}\right\}$ for any $v_{1}, v_{2} \in S$ contains $K_{5}^{-}$as a subgraph.

Lemma 2.7 Let $G$ be a graph with $|G| \in\{7, \ldots, 11\}$ and $\delta(G) \geq 6$. Then either $G$ contains $K_{5}$ as a subgraph, or for any set $T \subseteq V(G)$ with $|T|=5$ there exists $v \in T$ such that $G-v$ has a $K_{4}$ minor rooted at $T \backslash\{v\}$.

Proof. By Lemma 2.6, if $G$ is not 4-connected, then $G$ contains $K_{5}$ as a subgraph and we are done. Thus we may assume $G$ is 4 -connected. Let $T \subseteq V(G)$ such that $|T|=5$. Assume first that $G$ is 5 -connected, and let $v \in T$ be arbitrary. Then $G-v$ is a 4 -connected graph on $|G|-1$ vertices with $\delta(G) \geq 5$. Note that $e(G-v) \geq\left\lceil\frac{1}{2}(|G|-1) \delta(G)\right\rceil \geq 3(|G|-1)-6$, and it follows from Theorem 2.5 that $G-v$ has a $K_{4}$ minor rooted at $T \backslash\{v\}$. Therefore we may assume $G$ is not 5-connected, and so by Lemma $2.6 G$ contains $K_{5}^{-}$as a subgraph. In particular, $G$ contains a $K_{4}$ subgraph $H$. Since $G$ is 4-connected, there exist four disjoint paths $P_{1}, P_{2}, P_{3}, P_{4}$ with one end in $V(H)$, one end in $T$, and no internal vertices in $V(H) \cup T$. Let $v \in T$ be the unique vertex not met by any $P_{i}$. Then contracting each path $P_{i}$ to a single vertex gives a $K_{4}$ minor rooted at $T$ in $G-v$.

Lemma 2.8 Let $G$ be a graph with $|G| \in\{8, \ldots, 11\}$ and $\delta(G) \geq 6$, and let $v_{1}, \ldots, v_{6} \in$ $V(G)$ be distinct. If $v_{1} v_{2} \notin E(G)$, then there exists some component $C$ of $G-\left\{v_{1}, \ldots, v_{6}\right\}$ such that either $\left\{v_{1}, v_{2}\right\} \subseteq N_{G}(C)$ or $\left\{v_{3}, \ldots, v_{6}\right\} \subseteq N_{G}(C)$.

Proof. Suppose for a contradiction that no such component exists. Put $T=\left\{v_{1}, \ldots, v_{6}\right\}$. 


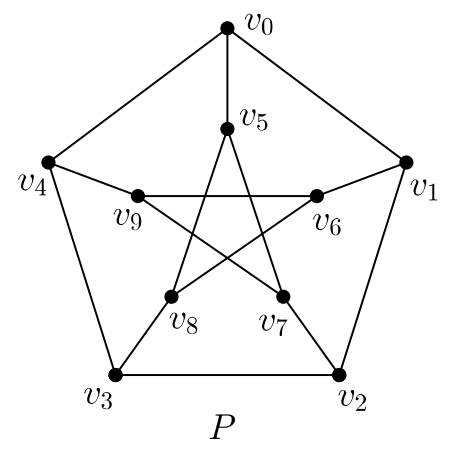

Figure 2.1: The Petersen graph $P$.

Since $\delta(G) \geq 6$ and $|T|=6$, every vertex of $T$ has at least one neighbor in $V(G) \backslash T$. Thus it must be the case that $G-T$ is disconnected. Let $C_{1}, C_{2}$ be distinct components of $G-T$. Then for $i \in\{1,2\}$, any vertex of $C_{i}$ is adjacent to at most four vertices of $T$, and it follows that $\left|V\left(C_{i}\right)\right| \geq 3$ since $\delta(G) \geq 6$. But then $|G| \geq|T|+\left|C_{1}\right|+\left|C_{2}\right| \geq 12$, a contradiction.

The following lemma is proved by computer search. The details of this search can be found in [14].

Lemma 2.9 If $G$ is a graph with $|G| \in\{9,10,11\}$ and $\delta(G) \geq 6$, then either $G$ contains $K_{7}^{=} \cup K_{1}$ as a minor, or $G$ is isomorphic to one of the five graphs $\overline{C_{5}} \vee \overline{C_{4}}, \overline{C_{9}}, K_{3,3,3}, \overline{C_{6}} \vee \overline{K_{3}}$, or $\bar{P}$, where $\bar{P}$ is the complement of the Petersen graph $P$. Furthermore, the graphs $\overline{C_{5}} \vee \overline{C_{4}}$ and $\overline{C_{9}}$ contain $K_{7}^{-}$as a minor, and the graphs $K_{3,3,3}, \overline{C_{6}} \vee \overline{K_{3}}$, and $\bar{P}$ are all edge maximal with respect to not having a $K_{7}^{=} \cup K_{1}$ minor.

The remaining results in this section will all be used in the proof of Theorem 1.2 to handle the counterexamples $K_{3,3,3}, \overline{C_{6}} \vee \overline{K_{3}}$, and $\bar{P}$ of Lemma 2.9 .

Lemma 2.10 Let $G$ be isomorphic to either $K_{3,3,3}$ or $\overline{C_{6}} \vee \overline{K_{3}}$. If $e_{1}, e_{2}, e_{3}$ are distinct missing edges of $G$ such that $e_{1}, e_{2}$ share a common end, then $G+\left\{e_{1}, e_{2}, e_{3}\right\} \geq K_{8}^{=}$.

Proof. Since $e_{1}, e_{2}, e_{3}$ are not independent, there exist $u, v \in V(G)$ such that $u v \in E(G)$ and $u, v$ are both incident to two missing edges of $G+\left\{e_{1}, e_{2}, e_{3}\right\}$. By contracting $u v$ we 
obtain a $K_{8}^{=}$minor of $G+\left\{e_{1}, e_{2}, e_{3}\right\}$.

Lemma 2.11 Let $e_{1}, e_{2}, e_{3}$ be three distinct missing edges of $\bar{P}$ such that no vertex of $\bar{P}$ is incident to every $e_{i}$. If either the $e_{i}$ do not all belong to the same 5 vertex cycle in $P$ or the $e_{i}$ induce a 4 vertex path in $P$, then $\bar{P}+\left\{e_{1}, e_{2}, e_{3}\right\} \geq K_{8}^{=}$. Furthermore, the graph obtained from $\bar{P}$ by adding any four missing edges contains $K_{8}^{=}$as a minor.

Proof. Assume first that $e_{1}, e_{2}, e_{3}$ all belong to a 4 vertex path in $P$. Without loss of generality, $\left\{e_{1}, e_{2}, e_{3}\right\}=\left\{v_{0} v_{1}, v_{1} v_{2}, v_{2} v_{3}\right\}$, where the vertices of $P$ are labeled as in Figure 2.1. By contracting the edges $v_{0} v_{6}$ and $v_{3} v_{7}$, we see that $\bar{P}+\left\{v_{0} v_{1}, v_{1} v_{2}, v_{2} v_{3}\right\} \geq K_{8}^{=}$. So we may now assume that $e_{1}, e_{2}, e_{3}$ do not all belong to some 5 vertex cycle in $P$. If the edges $e_{i}$ are pairwise disjoint, then without loss of generality, $\left\{e_{1}, e_{2}, e_{3}\right\}$ is one of the sets $\left\{v_{0} v_{5}, v_{2} v_{3}, v_{6} v_{9}\right\},\left\{v_{0} v_{1}, v_{2} v_{3}, v_{5} v_{8}\right\},\left\{v_{0} v_{5}, v_{1} v_{2}, v_{3} v_{4}\right\}$, or $\left\{v_{0} v_{5}, v_{1} v_{6}, v_{3} v_{4}\right\}$. In each case, it is straightforward to show that $\bar{P}+\left\{e_{1}, e_{2}, e_{3}\right\} \geq K_{8}^{=}$. So we may assume that two $e_{i}$ are incident to the same vertex, say $\left\{e_{1}, e_{2}\right\}=\left\{v_{0} v_{1}, v_{0} v_{4}\right\}$. Then $e_{3}$ is either incident to $v_{5}$ or not, and by symmetry we may assume $e_{3}$ is either $v_{5} v_{7}$ or $v_{6} v_{8}$. Both of these cases are also straightforward to verify. For the second part of the statement, it is easy to see that given any four missing edges of $\bar{P}$, some three of those edges satisfy the conditions of the first part of the statement.

Lemma 2.12 Let $A_{1}, A_{2} \subseteq V(\bar{P})$ such that $\left|A_{1}\right| \geq\left|A_{2}\right| \geq 7$. Then for $i \in\{1,2\}$ there exist vertices $v_{i} \in A_{i}$ such that the graph obtained from $\bar{P}$ by adding all missing edges incident to $v_{i}$ in $\bar{P}\left[A_{i}\right]$ contains $K_{8}^{=}$as a minor.

Proof. Let the vertices of $P$ be as labeled in Figure 2.1. Since $\left|A_{1}\right| \geq 7$, there must exist two missing edges of $\bar{P}\left[A_{1}\right]$ which share a common end, say $\left\{v_{0}, v_{1}, v_{4}\right\} \subseteq A_{1}$. From Lemma 2.11, it follows that if $\bar{P}\left[A_{2}\right]$ contains any missing edge $e$ other than $v_{0} v_{1}, v_{0} v_{4}, v_{0} v_{5}, v_{2} v_{3}$, or $v_{6} v_{9}$, 
then we are done by adding all missing edges in $\bar{P}\left[A_{1}\right]$ incident to $v_{0}$ and all missing edges in $\bar{P}\left[A_{2}\right]$ incident to one end of $e$. Since $\left|A_{2}\right| \geq 7$, such an edge $e$ must exist.

\section{Proof of Theorem 1.2}

Suppose that $G$ is a minimum counterexample to Theorem 1.2 , and put $n:=|G|$. Then $e(G) \geq 6 n-20$, but $G$ is not a $\left(K_{8}, K_{2,2,2,2,2}, 5\right)$-cockade and $G \nsupseteq K_{9}^{=}$. We may suppose that $e(G)=6 n-20$. It is easy to verify that $n \geq 11$.

(1) $\delta(G) \geq 7$.

Let $x \in V(G)$ such that $d_{G}(x)=\delta(G)$. Then $e(G-x)=6 n-20-\delta(G)=6(n-1)-14-$ $\delta(G)$. If $e(G-x)>6(n-1)-20$, then by the minimality of $G$ and Lemma $2.4, G-x \geq K_{9}^{=}$, a contradiction since $G \geq G-x$. If $e(G-x)=6(n-1)-20$, then $d_{G}(x)=6$, and since $G \nsupseteq K_{9}^{=}$, we see by the minimality of $G$ that $G-x$ is a $\left(K_{8}, K_{2,2,2,2,2}, 5\right)$-cockade. But then by Lemma 2.2, $G \geq K_{9}^{=}$, a contradiction. Hence, $e(G-x)<6(n-1)-20$. It follows that $\delta(G)>6$.

(2) $\delta(G[N(x)]) \geq 6$ for all $x \in V(G)$.

Let $x y \in E(G)$ and put $d:=|N(x) \cap N(y)|$. Then $e(G / x y)=6 n-20-(d+1)=$ $6(n-1)-15-d$. If $e(G / x y)>6(n-1)-20$, then by Lemma 2.4 and the minimality of $G$, $G / x y \geq K_{9}^{=}$, a contradiction since $G \geq G / x y$. If $e(G / x y)=6(n-1)-20$, then $d=5$, and since $G \nsupseteq K_{9}^{=}$, we see by the minimality of $G$ that $G / x y$ is a $\left(K_{8}, K_{2,2,2,2,2}, 5\right)$-cockade. But then by Lemma 2.3 and (1), $G \geq K_{9}^{=}$, a contradiction. Hence, $e(G / x y)<6(n-1)-20$. It follows that $d>5$.

We will utilize the following notation throughout the remainder of the proof. Let $S$ be a minimal separating set in $G$, and let $G_{1}$ and $G_{2}$ be two subgraphs of $G$ such that $G=G_{1} \cup G_{2}$ and $G_{1} \cap G_{2}=G[S]$. It is an immediate consequence of (1) that 
(3) $\left|G_{i}\right| \geq 8$ for $i \in\{1,2\}$.

(4) Neither $G_{1}$ nor $G_{2}$ is a $\left(K_{8}, K_{2,2,2,2,2}, 5\right)$-cockade.

Suppose that $G_{1}$, say, is a $\left(K_{8}, K_{2,2,2,2,2}, 5\right)$-cockade. If $|S| \geq 6$, then by contracting any component of $G_{2}-S$ to a single vertex, it follows from Lemma 2.2 that $G \geq K_{9}^{=}$, a contradiction. Hence $|S| \leq 5$, and it follows from Lemma 2.1 that $G[S]$ is complete. Then since $e\left(G_{2}\right)=e(G)-e\left(G_{1}\right)+e(G[S])$, from Lemma 2.4 we have $e\left(G_{2}\right)=(6 n-20)-\left(6\left|G_{1}\right|-\right.$ $20)+\left(\begin{array}{c}|S| \\ 2\end{array}\right)=6\left|G_{2}\right|-6|S|+\left(\begin{array}{c}|S| \\ 2\end{array}\right)$. If $|S| \leq 4$, then $e\left(G_{2}\right) \geq 6\left|G_{2}\right|-18$, and it follows from (3), Lemma 2.4, and the minimality of $G$ that $G_{2} \geq K_{9}^{=}$, a contradiction. Hence $|S|=5$, and we have $e\left(G_{2}\right)=6\left|G_{2}\right|-20$. Since $G \nsupseteq K_{9}^{=}$, we see $G_{2} \nsupseteq K_{9}^{=}$, and so by (3) and the minimality of $G, G_{2}$ is a $\left(K_{8}, K_{2,2,2,2,2}, 5\right)$-cockade. However, since $G[S]$ is isomorphic to $K_{5}$, it follows that $G$ is also a $\left(K_{8}, K_{2,2,2,2,2}, 5\right)$-cockade, a contradiction.

For $i \in\{1,2\}$, let $d_{i}$ be the maximum number of edges that can be added to $G_{3-i}$ by contracting edges of $G$ with at least one end in $G_{i}$. More precisely, let $d_{i}$ be the largest integer such that $G_{i}$ contains disjoint sets of vertices $V_{1}, V_{2}, \ldots, V_{|S|}$ so that $G_{i}\left[V_{j}\right]$ is connected and $\left|S \cap V_{j}\right|=1$ for $j \in\{1, \ldots,|S|\}$, and such that the graph obtained from $G_{i}$ by contracting $V_{1}, V_{2}, \ldots, V_{p}$ each to a single vertex and deleting $V(G) \backslash\left(\cup_{j} V_{j}\right)$ has $e(G[S])+d_{i}$ edges. Let $G_{i}^{*}$ be a graph obtained from $G$ by contracting $G_{3-i}$ onto $S$ so that $G_{i}^{*}-S=G_{i}-S$ and $e\left(G_{i}^{*}\right)=e\left(G_{i}\right)+d_{3-i}$ for $i \in\{1,2\}$.

(5) For $i \in\{1,2\}, e\left(G_{i}\right) \leq 6\left|G_{i}\right|-20-d_{3-i}$, with equality only if $G_{i}^{*}$ is a $\left(K_{8}, K_{2,2,2,2,2}, 5\right)$ cockade.

If, say, $e\left(G_{1}\right)>6\left|G_{1}\right|-20-d_{2}$, then $e\left(G_{1}^{*}\right)>6\left|G_{1}^{*}\right|-20$, and so it follows from (3), Lemma 2.4, and the minimality of $G$ that $G_{1}^{*} \geq K_{9}^{=}$, a contradiction. If $e\left(G_{1}\right)=6\left|G_{1}\right|-20-$ $d_{2}$, then $e\left(G_{1}^{*}\right)=6\left|G_{1}^{*}\right|-20$, and then since $G \nsupseteq K_{9}^{=}$, it now follows from the minimality of $G$ that $G_{1}^{*}$ is a $\left(K_{8}, K_{2,2,2,2,2}, 5\right)$-cockade.

(6) $e(G) \leq 6 n-40+6|S|-d_{1}-d_{2}-e(G[S])$. 
This follows from (5) and the fact that $e(G)=e\left(G_{1}\right)+e\left(G_{2}\right)-e(G[S])$.

(7) $G$ is 5-connected.

Since $e(G)=6 n-20$, it follows from (6) that $6|S| \geq 20+d_{1}+d_{2}+e(G[S])$. Now since $e(G[S]) \geq \frac{1}{2} \delta(G[S])|S|$ and $d_{i} \geq|S|-\delta(G[S])-1$ for $i \in\{1,2\}$, we see $4|S| \geq$ $18+\frac{1}{2} \delta(G[S])(|S|-4)$. Therefore $|S| \geq 5$.

(8) If $|S| \in\{5,6\}$, then for $i \in\{1,2\}$ there exists $x \in V\left(G_{i}\right) \backslash S$ with $d_{G}(x) \leq 11$.

Suppose to the contrary that $d_{G}(x) \geq 12$ for every vertex $x \in V\left(G_{1}\right) \backslash S$, say. Then $\left|G_{1}\right| \geq$ 13 and $\left|G_{1}\right|-|S| \geq 7$. Then $2 e\left(G_{1}\right) \geq 12\left(\left|G_{1}\right|-|S|\right)+e\left(V\left(G_{1}\right) \backslash S, S\right)+2 e(G[S])$. By (5), we also have $2 e\left(G_{1}\right) \leq 12\left|G_{1}\right|-40-2 d_{2}$. Hence $e\left(V\left(G_{1}\right) \backslash S, S\right) \leq 12|S|-40-2\left(d_{2}+e(G[S])\right)$. Now there exists $y_{1} \in V\left(G_{2}\right) \backslash S$. Since $|S| \leq 6$ and $d_{G}\left(y_{1}\right) \geq 7$ by $(1), y_{1}$ has a neighbor $y_{2} \in V\left(G_{2}\right) \backslash S$. By $(2), y_{1}$ and $y_{2}$ have at least 6 common neighbors. Say $y_{3}, y_{4}, \ldots, y_{|S|}$ are common neighbors of $y_{1}$ and $y_{2}$ in $G$. Note that $N\left(y_{1}\right) \subseteq V\left(G_{2}\right)$. Now there exist disjoint paths $P_{1}, \ldots, P_{|S|}$ in $G$, each with one end in $\left\{y_{1}, \ldots, y_{|S|}\right\}$, the other end in $S$, and all internal vertices in $G_{2}-S$. By contracting each of these paths onto its end in $S$, it follows that $e(G[S])+d_{2} \geq 2(|S|-2)+1=2|S|-3$. Thus $e\left(V\left(G_{1}\right) \backslash S, S\right) \leq 8|S|-34$. Therefore $2 e\left(G_{1}-S\right) \geq 12\left(\left|G_{1}\right|-|S|\right)-e\left(V\left(G_{1}\right) \backslash S, S\right) \geq 12\left(\left|G_{1}\right|-|S|\right)-8|S|+34$, and so $e\left(G_{1}-S\right) \geq 6\left(\left|G_{1}\right|-|S|\right)-7$ since $|S| \in\{5,6\}$. Since $e\left(G_{1}-S\right) \leq\left(\begin{array}{c}\left|G_{1}\right|-|S| \\ 2\end{array}\right)$, this implies $\left|G_{1}\right|-|S| \geq 12$. From the minimality of $G$ and Lemma 2.4 , we see $G_{1}-S \geq K_{9}^{=}$, a contradiction.

(9) $G$ is 6-connected.

Suppose not. By (7), there exists a minimal separating set $S$ of $G$ with $|S|=5$. By (8), there exists $x \in V\left(G_{1}\right) \backslash S$ such that $d_{G}(x) \leq 11$. By $(1), d_{G}(x) \geq 7$, and by $(2), \delta(G[N(x)]) \geq$ 6. If $G[N(x)]$ contains a subgraph $H$ isomorphic to $K_{5}$, then let $P_{1}, \ldots, P_{5}$ be disjoint paths in $G$ with one end in $V(H)$ and one end in $S$. Otherwise, let $P_{1}, \ldots, P_{5}$ be disjoint paths with one end in $N(x)$, the other end in $S$, and no internal vertices in $N(x)$. Say $V\left(P_{i}\right) \cap N(x)=v_{i}$ 
for $i \in\{1, \ldots, 5\}$. Then by Lemma 2.7 the end of some $P_{i}$ in $N(x)$, say $v_{1}$, is such that $G[N(x)]-v_{1}$ has a $K_{4}$ minor rooted at $\left\{v_{2}, v_{3}, v_{4}, v_{5}\right\}$. By additionally contracting the edge $x v_{1}$ if necessary, we may thus in both cases assume that the ends of $P_{1}, \ldots, P_{5}$ in $N(x)$ belong to a $K_{5}$ subgraph of some minor of $G[N[x]]$. By now contracting each path $P_{i}$ onto its end in $S$, it follows that $d_{1}+e(G[S])=10$. Similarly, $d_{2}+e(G[S])=10$, and so $d_{1}=d_{2}$. Note that by (4) and (5), for $i \in\{1,2\}$ we must have either $d_{3-i} \geq 1$ or $e\left(G_{i}\right) \leq 6\left|G_{i}\right|-21-d_{3-i}$. Then in either case, $6 n-20=e(G)=e\left(G_{1}\right)+e\left(G_{2}\right)-e(G[S]) \leq$ $6 n-10-\max \left\{1, d_{1}\right\}-\left(d_{2}+e(G[S])\right) \leq 6 n-21$, a contradiction.

(10) There is no minimal separating set $S$ of $G$ such that $G[S]$ is complete.

Suppose such a separating set $S$ exists. If $|S| \geq 7$, then by contracting any two components of $G-S$ to a single vertex each, we see $G \geq K_{9}^{-}$, a contradiction. Thus, by (9), $|S|=6$. Since $G[S]$ is complete, we have $d_{1}=d_{2}=0$. For $i \in\{1,2\}$, since $G_{i} \ngtr K_{9}^{=}$and $G_{i}$ is not a $\left(K_{8}, K_{2,2,2,2,2}, 5\right)$-cockade by (4), we have $e\left(G_{i}\right) \leq 6\left|G_{i}\right|-21$ by (5). But then $6 n-20=e(G)=e\left(G_{1}\right)+e\left(G_{2}\right)-e(G[S]) \leq\left(6\left|G_{1}\right|-21\right)+\left(6\left|G_{2}\right|-21\right)-15=6 n-21, \mathrm{a}$ contradiction.

(11) $\delta(G) \geq 8$.

Suppose there exists $x \in V(G)$ such that $d_{G}(x) \leq 7$. By $(1), d_{G}(x)=7$. By (2), $\delta(G[N(x)])=6$, and so $G[N(x)]$ is isomorphic to $K_{7}$. But $N(x)$ is a separating set of $G$, and so contains some minimal separating set of $G$, contrary to (10).

(12) There is no minimal separating set $S$ of $G$ such that $G[S \backslash\{x\}]$ is complete for some $x \in S$.

Suppose there exists $x \in S$ such that $G[S \backslash\{x\}]$ is complete. Note that $|S| \geq 6$ by (9). If $|S| \geq 8$, then since $G[S \backslash\{x\}]$ contains $K_{7}$ as a subgraph, we can contract any two components of $G-S$ each to a single vertex to obtain a $K_{9}^{-}$minor, a contradiction. So $|S| \in\{6,7\}$. By contracting any component of $G_{i}-S$ onto $x$, we see that $d_{i}+e(G[S])=\left(\begin{array}{c}|S| \\ 2\end{array}\right)$ for $i \in\{1,2\}$, 
and in particular $d_{1}=d_{2}=|S|-\delta(G[S])-1 \geq 1$. Suppose, say, $e\left(G_{1}\right) \leq 6\left|G_{1}\right|-21-d_{2}$. Then $6 n-20=e(G)=e\left(G_{1}\right)+e\left(G_{2}\right)-e(G[S]) \leq\left(6\left|G_{1}\right|-21-d_{2}\right)+\left(6\left|G_{2}\right|-20-d_{1}\right)-e(G[S]) \leq$ $6 n-21$, a contradiction.

Hence by (5), we have $e\left(G_{i}\right)=6\left|G_{i}\right|-20-d_{3-1}$, and $G_{i}^{*}$ is a $\left(K_{8}, K_{2,2,2,2,2}, 5\right)$-cockade for $i \in\{1,2\}$. Now $6 n-20=e(G)=e\left(G_{1}\right)+e\left(G_{2}\right)-e(G[S])=\left(6\left|G_{1}\right|-20-d_{2}\right)+\left(6\left|G_{2}\right|-20-\right.$ $\left.d_{1}\right)-e(G[S])=6 n-19-d_{1}$. Thus we must have $d_{1}=d_{2}=1$, and so $G[S]$ is isomorphic to $K_{|S|}^{-}$. Since $G_{i}^{*}[S]$ contains a $K_{6}$ subgraph, there must exist some $K_{8}$ subgraph $H_{i}$ of $G_{i}^{*}$ such that $S \subseteq V\left(H_{i}\right)$ for $i \in\{1,2\}$. Then $G\left[V\left(H_{i}\right)\right]$ is isomorphic to $K_{8}^{-}$. Thus $G\left[V\left(H_{1} \cup H_{2}\right)\right]$ is not a $\left(K_{8}, K_{2,2,2,2,2}, 5\right)$-cockade, but is a graph on $16-|S|$ vertices with $6(16-|S|)-20$ edges. Since $n \geq 11>16-|S|, G\left[V\left(H_{1} \cup H_{2}\right)\right]$ contains a $K_{9}^{=}$minor by the minimality of $G$, a contradiction.

(13) $\delta(G) \geq 9$.

Suppose there exists $x \in V(G)$ such that $d_{G}(x) \leq 8$. By $(11), d_{G}(x)=8$. Since $\delta(G[N(x)]) \geq 6$ by (2), it follows that $G[N(x)]$ is isomorphic to $K_{8}-M$, where $M$ is a matching of $N(x)$. If $|M| \leq 2$, then $G[N[x]]$ contains $K_{9}^{=}$as a subgraph, a contradiction. Suppose $|M|=3$. Let $C$ be a component of $G-N[x]$. Since $G$ is 6 -connected by $(9), N(C)$ contains both ends of some missing edge $e$ of $G[N(x)]$. By contracting $C$ onto one end of $e$, we see that $G \geq K_{9}^{=}$, a contradiction. Hence $|M|=4$. That is, $G[N(x)]$ is isomorphic to $K_{2,2,2,2}$. Say $N(x)=\left\{v_{1}, w_{1}, \ldots, v_{4}, w_{4}\right\}$, where $v_{i} w_{i} \notin E(G)$ for $i \in\{1, \ldots, 4\}$.

(13.1) $G-N[x]$ is connected.

If not, let $C_{1}, C_{2}$ be distinct components of $G-N[x]$. Since $G$ is 6 -connected by (9), $N\left(C_{1}\right)$ and $N\left(C_{2}\right)$ each contain at least two nonadjacent pairs of vertices of $N(x)$. Thus it is possible to pick distinct pairs from each of $N\left(C_{1}\right)$ and $N\left(C_{2}\right)$, say $v_{i}, w_{i} \in N\left(C_{i}\right)$ for $i \in\{1,2\}$. By contracting $C_{i}$ onto $v_{i}$ for $i \in\{1,2\}$ we see $G \geq K_{9}^{=}$, a contradiction.

(13.2) No vertex of $G-N[x]$ is adjacent to a pair of nonadjacent vertices of $N(x)$. 
Suppose to the contrary that there exists $z \in V(G) \backslash N[x]$ such that $z$ is adjacent to, say, $v_{1}$ and $w_{1}$. Let $C$ be a component of $G-N[x]-z$. If $N(C)$ contains some pair of nonadjacent vertices of $N(x)$ distinct from $v_{1}, w_{1}$, say $v_{2}, w_{2} \in N(C)$, then by contracting $C$ onto $v_{2}$ and contracting the edge $v_{1} z$, we see $G \geq K_{9}^{=}$, a contradiction. Hence $N(C)$ can contain no nonadjacent pair of vertices of $N(x)$ other than $v_{1}, w_{1}$. It follows that $|N(C) \cap N(x)| \leq 5$. Since $G$ is 6 -connected by (9), we conclude $|N(C) \cap N(x)|=5$, and $\left\{v_{1}, w_{1}, z\right\} \subseteq N(C)$. Without loss of generality, $N(C)=\left\{v_{1}, v_{2}, v_{3}, v_{4}, z, w_{1}\right\}$. Since $N(C)$ is a minimal separating set of $G$, we may put $S=N(C)$. Note that $G\left[\left\{v_{1}, w_{1}, v_{2}, v_{3}, v_{4}\right\}\right]$ is isomorphic to $K_{5}^{-}$, so $e(G[S]) \geq 11$. By $(8)$, there exists $y \in V\left(G_{1}\right) \backslash S$ such that $d_{G}(y) \leq 11$. Since $G$ is 6 -connected by (9), there exist disjoint paths $P_{1}, \ldots, P_{6}$ with one end in $N(y)$, the other end in $S$, and no internal vertices in $N(y) \cup S$. Say $u_{i}$ is the end of $P_{i}$ in $N(y)$ for $i \in\{1, \ldots, 6\}, v_{i}$ is the end of $P_{i}$ in $S$ for $i \in\{1, \ldots, 4\}$, and $P_{5}, P_{6}$ have ends $z, w_{1}$ in $S$, respectively. If $u_{1} u_{6} \in E(G)$, then by contracting each path $P_{i}$ onto its end in $S$ and additionally contracting the edge $y u_{5}$, we see $e(G[S])+d_{2}=15$. Otherwise, by Lemma 2.8 there exists some component $C^{\prime}$ of $G[N(y)]-\left\{u_{1}, \ldots, u_{6}\right\}$ such that either $\left\{u_{1}, u_{6}\right\} \subseteq N\left(C^{\prime}\right)$ or $\left\{u_{2}, \ldots, u_{5}\right\} \subseteq N\left(C^{\prime}\right)$. By contracting $C^{\prime}$ onto $u_{1}$ and contracting $y u_{5}$ in the former case, or contracting $C^{\prime}$ onto $u_{5}$ and contracting $y u_{1}$ in the latter case, and then contracting each path $P_{i}$ onto its end in $S$ we again see $e(G[S])+d_{2}=15$. By symmetry, $e(G[S])+d_{1}=15$ as well. By (12), $d_{i} \geq 2$ for $i \in\{1,2\}$. Thus by $(6)$, we see $6 n-20=e(G) \leq 6 n-40+36-\left(e(G[S])+d_{2}\right)-d_{1} \leq 6 n-21$, a contradiction.

(13.3) Every vertex of $N(x)$ has at least three neighbors in $G-N[x]$.

Since $\delta(G) \geq 8$ by (11), there exists $z \in V(G) \backslash N[x]$ such that $z v_{1} \in E(G)$, say. By (2), $z$ and $v_{1}$ have at least six common neighbors. By (13.2), at most three of these common neighbors can belong to $N(x)$, and (13.3) follows.

(13.4) $G-N[x]$ is 2-connected. 
By (13.1), suppose to the contrary that $z$ is a cut-vertex of $G-N[x]$. If $C_{1}$ and $C_{2}$ are components of $G-N[x]-z$ with, say, $v_{i}, w_{i} \in N\left(C_{i}\right)$ for $i \in\{1,2\}$, then by contracting each $C_{i}$ onto $v_{i}$ we see $G \geq K_{9}^{=}$, a contradiction. Since $G$ is 6 -connected by (9), it follows that every component $C$ of $G-N[x]-z$ satisfies $|N(C) \cap N(x)|=5$, with $v_{1}$ and $w_{1}$, say, in $N(C)$, and at most one of $v_{i}$ or $w_{i}$ in $N(C)$ for $i \in\{2,3,4\}$. Without loss of generality, assume $C_{1}$ is a component of $G-N[x]-z$ with $\left\{v_{2}, v_{3}, v_{4}\right\} \subseteq N(C)$. Consider the three edges $e_{1}=v_{2} w_{3}, e_{2}=v_{3} w_{2}$, and $e_{3}=w_{2} w_{3}$. Then for $i \in\{1,2,3\}$, by (2) the ends of $e_{i}$ have at least six common neighbors, only five of which belong to $N[x]$, and so there exists $y_{i} \in V(G) \backslash N[x]$ adjacent to both ends of $e_{i}$. By (13.2), $y_{1}, y_{2}, y_{3}$ are distinct, and in particular at most one $y_{i}=z$. As $w_{2}, w_{3} \notin N\left(C_{1}\right), y_{i} \notin V\left(C_{1}\right)$ for $i \in\{1,2,3\}$. Furthermore, if two of $y_{1}, y_{2}, y_{3}$ belong to the same component $C_{2}$ of $G-N[x]-z$, then $C_{2}$ is a component with $v_{2}, w_{2} \in N\left(C_{2}\right)$, say, contrary to the above. Thus there exist at least two components $C_{2}$ and $C_{3}$ of $G-N[x]-z$ distinct from $C_{1}$. Without loss of generality, we may assume $w_{2} \in N\left(C_{2}\right)$. Now by contracting $C_{1}$ and $C_{2}$ onto $z$, contracting $C_{3}$ onto $v_{1}$, and contracting the edge $v_{2} z$, we see $G \geq K_{9}^{=}$, a contradiction.

We now consider the graph $H=G-\left\{x, v_{3}, w_{3}, v_{4}, w_{4}\right\}$. We claim that $H$ is 4-connected. Suppose $Q$ is a minimum separating set of at most three vertices in $H$. By (13.4), we have $|Q| \geq 2$ and $|Q \cap N(x)| \leq 1$. If $|Q \cap N(x)|=1$, then by symmetry we may assume $w_{2} \in Q$. Since $H\left[\left\{v_{1}, w_{1}, v_{2}\right\}\right]$ is connected, $v_{1}, w_{1}$, and $v_{2}$ all belong to the same component $C$ of $H-Q$. If $w_{2} \notin Q$, then $w_{2}$ also belongs to $C$, and in this case we assume that $Q$ and $w_{2}$ are chosen so that $\left|Q \cap N\left(w_{2}\right)\right|$ is maximal. We next claim that there exist $v_{1}^{\prime}$ and $w_{1}^{\prime}$ in $V(G) \backslash(N[x] \cup Q)$ adjacent to $v_{1}$ and $w_{1}$, respectively. If not, then by (13.2) and (13.3), it must be the case that $w_{1}$, say, has exactly three neighbors $z_{1}, z_{2}, z_{3}$ in $G-N[x]$, and $Q=\left\{z_{1}, z_{2}, z_{3}\right\}$. Now $w_{2} \notin Q$, so by our choice of $Q$ and $w_{2}$, it follows that $w_{2}$ is complete to $Q$. Since $v_{2} w_{1} \in E(G), v_{2}$ and $w_{1}$ have at least one common neighbor in $G-N[x]$ by $(2)$. This common neighbor must be one of $z_{1}, z_{2}, z_{3}$, say $z_{1}$, but then $z_{1}$ is adjacent to $v_{2}$ and $w_{2}$, 
contradicting (13.2). Thus the claim is proved, and there exist $v_{1}^{\prime}, w_{1}^{\prime} \in V(G) \backslash(N[x] \cup Q)$ such that $v_{1} v_{1}^{\prime}, w_{1} w_{1}^{\prime} \in E(G)$. Now we have $v_{1}^{\prime}, w_{1}^{\prime} \in V(C)$. By (13.4), there exist two internally disjoint $v_{1}^{\prime}, w_{1}^{\prime}$-paths in $G-N[x]$. Since $|Q| \leq 3$, at least one of these paths must be contained entirely within $G[V(C) \cup Q]$. Note that since $G \nsupseteq K_{9}^{=}$, there must then be no $v_{i}, w_{i}$-path in $G\left[V\left(C^{\prime}\right) \cup\left\{v_{i}, w_{i}\right\}\right]$ for $i \in\{3,4\}$, where $C^{\prime}$ is any component of $H-Q$ distinct from $C$. Hence at most one of $v_{i}, w_{i}$ has a neighbor in $C^{\prime}$ for $i \in\{3,4\}$. It follows that $C^{\prime}$ is separated from $x$ by $Q$ and at most two vertices of $N(x)$. But since $|Q| \leq 3$, this contradicts that $G$ is 6 -connected by (9). This proves the claim that $H$ is 4 -connected.

If there exists a $K_{4}$ minor of $H$ rooted at $\left\{v_{1}, w_{1}, v_{2}, w_{2}\right\}$, then $G \geq K_{9}^{=}$, a contradiction. Thus $e(H) \leq 3|H|-7=3(n-5)-7$ by Theorem 2.5. For $i \in\{3,4\}, v_{i}$ and $w_{i}$ have no common neighbor in $G-N[x]$ by (13.2), so they together have at most $|G|-|N[x]|=n-9$ neighbors in $G-N[x]$. Furthermore, the vertices $v_{3}, w_{3}, v_{4}, w_{4}$ are together incident with 20 edges of $G[N(x)]$. Therefore $6 n-20=e(G) \leq d_{G}(x)+20+2(n-9)+e(H) \leq 8+20+$ $2(n-9)+3(n-5)-7=5 n-12$. It follows that $n \leq 8$, a contradiction which completes the proof of (13).

(14) Suppose $x \in V(G)$ with $d_{G}(x) \in\{9,10,11\}$, and let $M \subseteq N(x)$ be the vertices of $N(x)$ which are not complete to all other vertices of $N(x)$. Then there is no component $C$ of $G-N[x]$ such that $N\left(C^{\prime}\right) \cap M \subseteq N(C)$ for all components $C^{\prime}$ of $G-N[x]$.

Suppose to the contrary that such a component $C$ exists. Among all vertices $x$ with $d_{G}(x) \in\{9,10,11\}$ for which such a component $C$ exists, choose $x$ to be of minimum degree. Note that $N\left(C^{\prime}\right) \cap M \neq \emptyset$ for all components $C^{\prime}$ of $G-N[x]$ by (10). Suppose for a contradiction that $M \backslash N(C) \neq \emptyset$, and choose $y \in M \backslash N(C)$ to be of minimum degree among all vertices in $M \backslash N(C)$. Then $d_{G}(y)<d_{G}(x)$ since $y$ has no neighbor outside $N[x]$ by the existence of $C$. Now let $K$ be the component of $G-N[y]$ containing $C$. We claim that $N(x) \backslash N[y] \nsubseteq V(K)$. So suppose instead that $N(x) \backslash N[y] \subseteq V(K)$. Let $C^{\prime}$ be any component of $G-N[x]$ distinct from $C$. Note that such a component $C^{\prime}$ exists since 
otherwise $K$ is the only component of $G-N[y]$, contrary to our choice of $x$ and $C$. Let $K^{\prime}$ be the component of $G-N[y]$ containing $C^{\prime}$. We may assume $C^{\prime}$ is chosen such that $K^{\prime} \neq K$, since otherwise $K$ is the only component of $G-N[y]$, again a contradiction. Then $V\left(K^{\prime}\right) \cap(N(x) \backslash N[y])=\emptyset$, since $N(x) \backslash N[y] \subseteq V(K)$. Hence $N\left(K^{\prime}\right)=N\left(C^{\prime}\right) \subseteq N(y)$. Thus we have that $N\left(C^{\prime}\right) \cap M \subseteq N(C) \cap N(y)$. Therefore $N\left(K^{\prime}\right) \cap M_{y} \subseteq N(K)$, where $M_{y}$ is the set of vertices of $N(y)$ not complete to all other vertices of $N(y)$. Noticing that $M_{y} \subseteq M$ and that the component $K^{\prime}$ was essentially arbitrary (every component $K^{\prime}$ of $G-N[y]$ corresponds with some component $C^{\prime}$ of $G-N[x]$ ), we see that the existence of $y$ and $K$ contradicts the choice of $x$ and $C$. Therefore $N(x) \backslash N[y] \nsubseteq Z V(K)$, as claimed.

Hence there exists some component $H$ of $G[N(x) \backslash N[y]]$ with $V(H) \cap N(C)=\emptyset$. We must have $d_{G}(z) \geq d_{G}(y)$ for all $z \in V(H)$ by the choice of $y$. If $|H|=1$, then it follows that $d_{G}(z)=d_{G}(y)$ and $N(z)=N(y)$. But then $H$ is a component of $G-N[y]$ contradicting the choice of $x$ and $C$. Thus $|H| \geq 2$. On the other hand, $|H| \leq d_{G}(x)-d_{G}(y) \leq$ $11-9=2$, and so $|H|=2, d_{G}(x)=11$, and $d_{G}(y)=9$. By (2) applied to $y$, we see that $G[N(y) \cap N(x)]$ has minimum degree at least 5. Then the edges of $G[N(x)]$ are the edges of $N(x) \cap N(y)$, edges incident with $y$, and edges incident with $V(H)$. Therefore, $e(G[N(x)]) \geq \frac{5}{2}\left(d_{G}(y)-1\right)+\left(d_{G}(y)-1\right)+\left(2\left(d_{G}(y)-1\right)-1\right)=43>5 d_{G}(x)-14$. By Theorem 1.1, we see that $G[N(x)] \geq K_{8}^{=}$, and therefore $G[N[x]] \geq K_{9}^{=}$, a contradiction. This proves that $M \backslash N(C)=\emptyset$, that is $M \subseteq N(C)$.

If $G[N(x)] \geq K_{7}=\cup K_{1}$, then let $y \in N(x)$ such that $G[N(x) \backslash\{y\}] \geq K_{7}^{\bar{~}}$. If $y \notin N(C)$, then $y$ is complete to $N(x) \backslash\{y\}$, and so $G[N(x)] \geq K_{8}^{=}$and $G[N[x]] \geq K_{9}^{=}$, a contradiction. Hence $y \in N(C)$ and every nonneighbor of $y$ in $N(x)$ also belongs to $N(C)$ since $M \subseteq N(C)$. Now by contracting $C$ onto $y$, we again find, along with $x$, a $K_{9}^{=}$minor in $G$, a contradiction. Hence by Lemma $2.9, G[N(x)]$ is isomorphic to one of the five graphs $\overline{C_{5}} \vee \overline{C_{4}}, \overline{C_{9}}, K_{3,3,3}$, $\overline{C_{6}} \vee \overline{K_{3}}$, or $\bar{P}$, where $\bar{P}$ is the complement of the Petersen graph. Suppose $G[N(x)]$ is isomorphic to one of $\overline{C_{5}} \vee \overline{C_{4}}$ or $\overline{C_{9}}$. In both cases, $G[N(x)] \geq K_{7}^{-}$and $N(x)=M \subseteq N(C)$, so 
by contracting $C$ to a single vertex we obtain, along with $x$, a $K_{9}^{=}$minor of $G$, a contradiction. Thus we may suppose $G[N(x)]$ is isomorphic to one of $K_{3,3,3}, \overline{C_{6}} \vee \overline{K_{3}}$, or $\bar{P}$. Note that by Lemma 2.9, these three graphs are edge-maximal subject to not having a $K_{7}^{=} \cup K_{1}$ minor. We first show the following.

(14.1) $G-N[x]$ is connected.

Suppose $C^{\prime}$ is a component of $G-N[x]$ distinct from $C$. By $(10), N\left(C^{\prime}\right)$ contains both ends of some missing edge $e$ of $G[N(x)]$, and we contract $C^{\prime}$ onto one end of $e$. Then

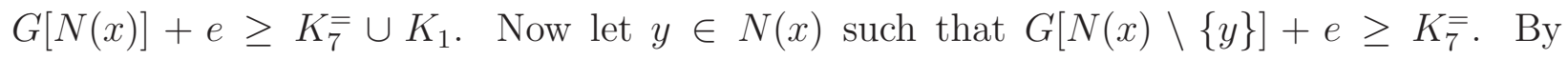
contracting $C$ onto $y$, we see $G \geq K_{9}^{=}$.

(14.2) $G[N(x)]$ is not isomorphic to either $K_{3,3,3}$ or $\overline{C_{6}} \vee \overline{K_{3}}$.

Say $N(x)=\left\{v_{1}, \ldots, v_{9}\right\}$ where $\left\{v_{1}, v_{2}, v_{3}\right\}$ is an independent set and either $\left\{v_{4}, v_{5}, v_{6}\right\}$ and $\left\{v_{7}, v_{8}, v_{9}\right\}$ are independent sets if $G[N(x)]$ is isomorphic to $K_{3,3,3}$ or $\left\{v_{4}, \ldots, v_{9}\right\}$ are the vertices of a $C_{6}$ in $\overline{G[N(x)]}$ written in cyclic order if $G[N(x)]$ is isomorphic to $\overline{C_{6}} \vee \overline{K_{3}}$. We claim that $G-N[x]$ is 3-connected. Suppose to the contrary that $Q$ is a minimum cut set of $G-N[x]$ with $|Q| \leq 2$. By (14.1), $|Q| \geq 1$. Let $C_{1}$ be any component of $G-(N[x] \cup Q)$, and let $C_{2}:=G-\left(N[x] \cup V\left(C_{1}\right)\right)$. Then $C_{2}$ is connected. Suppose that some vertex $v_{i} \in N(x)$ has no neighbor in $V\left(C_{2}\right)$. By (2), any neighbor of $v_{i}$ in $N(x)$ must have at least 2 neighbors in $V\left(C_{1}\right)$, that is, $\left\{v_{j} \in N(x): v_{i} v_{j} \in E(G)\right\} \subseteq N\left(C_{1}\right)$. In particular, there exist disjoint sets $T_{1}, T_{2} \subseteq N\left(C_{1}\right)$ such that $\left|T_{k}\right|=3$ and $G\left[T_{k}\right]$ contains at least two missing edges of $G[N(x)]$ for $k \in\{1,2\}$. Now since $C_{2}$ contains at least one component of $G-(N[x] \cup Q)$, it follows by (9) that $\left|N\left(C_{2}\right) \cap N(x)\right| \geq 4$. If $G\left[N\left(C_{2}\right)\right]$ contains some missing edge $e$ of $G[N(x)]$, then some $T_{k}$, say $T_{1}$, does not contain both ends of $e$. We contract $C_{2}$ onto one end of $e$, and we contract $C_{1}$ onto a vertex in $T_{1}$ incident to two missing edges of $G[N(x)]$, and from Lemma 2.10 we see, along with $x$, that $G \geq K_{9}^{=}$, a contradiction. If $G\left[N\left(C_{2}\right)\right]$ does not contain a missing edge of $G[N(x)]$, then it must be the case that $G[N(x)]$ is isomorphic to $\overline{C_{6}} \vee \overline{K_{3}},|Q|=2$, and $N\left(C_{2}\right) \cap N(x)=\left\{v_{3}, v_{4}, v_{6}, v_{8}\right\}$, 
say. Then there exist distinct $w_{1}, w_{2} \in V\left(C_{1}\right)$ such that $w_{1} v_{1}, w_{2} v_{2} \in E(G)$, and disjoint paths $P_{1}, P_{2}$ in $G-N[x]$ where $P_{i}$ has one end $w_{i}$ and one end in $Q$. Furthermore, since $C_{1}$ is connected there exists a path $P_{3}$ in $C_{1}$ with one end in $P_{1}$, the other end in $P_{2}$, and no internal vertices in $P_{1} \cup P_{2}$. Now by contracting $C_{2}$ onto $v_{3}$, contracting the paths $P_{i} \cup w_{i} v_{i}$ onto $v_{i}$ for $i \in\{1,2\}$, and contracting $P_{3}$ to a single edge, we see $G \geq K_{9}^{=}$by Lemma 2.10, a contradiction. Therefore we may assume that every vertex of $N(x)$ has some neighbor in $V\left(C_{2}\right)$. But now $\left|N\left(C_{1}\right) \cap N(x)\right| \geq 4$, and the same argument as above with the roles of $C_{1}$ and $C_{2}$ switched will give a contradiction. This establishes that $G-N[x]$ is 3-connected.

Note that since $\delta(G) \geq 9$ by (13), every vertex of $N(x)$ has at least two neighbors in $G-N[x]$. If some vertex $z \in V(G) \backslash N[x]$ is adjacent to both ends of a missing edge $e$ of $G[N(x)]$, then by contracting $G-N[x]-z$ onto a vertex of $N(x)$ incident to two missing edges of $G[N(x)]$ distinct from $e$, and then contracting $z$ onto one end of $e$, we get a $K_{9}^{=}$ minor by Lemma 2.10, a contradiction. Hence no vertex of $V(G) \backslash N[x]$ is adjacent to both ends of a missing edge of $G[N(x)]$. Thus we may select distinct $z_{1}, z_{2}, z_{3} \in V(G) \backslash N[x]$ such that $v_{i} z_{i} \in E(G)$ for $i \in\{1,2,3\}$. Since $G-N[x]$ is 3-connected, by an application of Menger's Theorem there exists a cycle in $G-N[x]$ containing all of $z_{1}, z_{2}, z_{3}$. By contracting each of the three subpaths of the cycle between the $z_{i}$ to a single edge, and then contracting each of the three edges $v_{i} z_{i}$, we again have $G \geq K_{9}^{=}$by Lemma 2.10. This contradiction proves (14.2).

Hence we may assume $G[N(x)]$ is isomorphic to $\bar{P}$, and we label the vertices of $\overline{G[N(x)]}$ as in Figure 2.1.

(14.3) No vertex of $G-N[x]$ is adjacent to both ends of a missing edge of $G[N(x)]$.

Suppose there exists $z \in V(G) \backslash N[x]$ adjacent to both ends of some missing edge $e$ of $G[N(x)]$, say $z v_{0}, z v_{1} \in E(G)$. Note that every vertex of $N(x)$ has at least two neighbors in $G-N[x]$ since $\delta(G) \geq 9$ by (13). Thus if $G-N[x]-z$ is connected, then by contracting $z$ onto $v_{0}$ and contracting $G-N[x]-z$ onto $v_{3}$, say, we add four edges to $G[N(x)]$, and 
it follows from Lemma 2.11 that $G \geq K_{9}^{=}$, a contradiction. So suppose $G-N[x]-z$ is disconnected. Since $G$ is 6 -connected by (9), we have $|N(C) \cap N(x)| \geq 5$ for all components $C$ of $G-N[x]-z$, and so $N(C)$ contains both ends of at least two missing edges of $G[N(x)]$. Let $C_{1}$ be a component of $G-N[x]-z$, and suppose $e_{1} \neq e$ is a missing edge of $G[N(x)]$ with both ends in $N\left(C_{1}\right)$. We consider three cases.

First, suppose $e$ and $e_{1}$ belong to a 6 -cycle, but not a 5 -cycle of $\overline{G[N(x)]}$, say $e_{1}=v_{7} v_{9}$. If $v_{2} \in N\left(C_{1}\right)$, then by contracting $C_{1}$ onto $v_{7}$ and contracting $z v_{0}$, we see $G \geq K_{9}^{=}$by Lemma 2.11, a contradiction. Hence $v_{2} \notin N\left(C_{1}\right)$, so there exists some component $C_{2}$ of $G-N[x]-z$ such that $v_{2} \in N\left(C_{2}\right)$. Now since $\left|N\left(C_{2}\right) \cap N(x)\right| \geq 5$, there must exist some missing edge $e_{2}$ of $G[N(x)]$ with both ends in $N\left(C_{2}\right)$ such that $e_{2}$ is distinct from $e$ and $e_{1}$. By contracting $C_{2}$ onto one end of $e_{2}, C_{1}$ onto $v_{7}$, and $z$ onto $v_{0}$, we again see $G \geq K_{9}^{=}$by Lemma 2.11, a contradiction.

Next, suppose $e$ and $e_{1}$ share a common end, say $e_{1}=v_{1} v_{2}$. If $v_{3} \in N\left(C_{1}\right)$, then by contracting $C_{1}$ onto $v_{2}$ and contracting $z v_{0}$, we see $G \geq K_{9}^{=}$by Lemma 2.11 , a contradiction. Thus there exists a component $C_{2} \neq C_{1}$ of $G-N[x]-z$ such that $v_{3} \in N\left(C_{2}\right)$. From Lemma 2.11, we may assume that $N\left(C_{2}\right)$ does not contain both ends of any missing edges of $G[N(x)]$ other than $v_{3} v_{4}, v_{5} v_{7}$, or $v_{1} v_{6}$. This requires $N\left(C_{2}\right) \cap N(x) \subseteq\left\{v_{1}, v_{3}, v_{4}, v_{5}, v_{6}, v_{7}\right\}$. By relabelling if necessary, we may assume $v_{3}, v_{4} \in N\left(C_{2}\right)$. By Lemma 2.11, we may assume that $N\left(C_{1}\right)$ does not contain both ends of any missing edges of $G[N(x)]$ other than $e_{1}$ or $v_{0} v_{1}$. This requires $N\left(C_{1}\right)=\left\{v_{0}, v_{1}, v_{2}, v_{8}, v_{9}, z\right\}$. Let $S=N\left(C_{1}\right)$. Then $S$ is a minimum separating set of $G$. Let $G_{1}$ and $G_{2}$ be as defined prior to (3), where we may assume $V\left(G_{1}\right) \backslash S=V\left(C_{1}\right)$. By contracting $V\left(C_{2}\right) \cup\left\{v_{3}, v_{4}\right\}$ onto $z$ and contracting $x v_{1}$, we see $e(G[S])+d_{2}=15$. By contracting $C_{1}$ onto $v_{1}$, we see $d_{1} \geq 2$. Thus by (6), we have $6 n-20=e(G) \leq 6 n-40+6 \cdot 6-2-15=6 n-21$, a contradiction.

Lastly, suppose $e$ and $e_{1}$ are disjoint and belong to a 5-cycle of $G[N(x)]$, say $e_{1}=v_{2} v_{3}$. If $v_{7} \in N\left(C_{1}\right)$, then by contracting $C_{1}$ onto $v_{2}$ and contracting $z v_{0}, G \geq K_{9}^{=}$by Lemma 2.11 , a 
contradiction. Thus there exists a component $C_{2} \neq C_{1}$ of $G-N[x]-z$ such that $v_{7} \in N\left(C_{2}\right)$. If any of $v_{2}, v_{5}$, or $v_{9}$ belongs to $N\left(C_{2}\right)$, it is now possible to contract $C_{1}, C_{2}$, and $z$ onto $N(x)$ such that $G \geq K_{9}^{=}$by Lemma 2.11 , a contradiction. So $N\left(C_{2}\right)$ contains at least four vertices of $\left\{v_{0}, v_{1}, v_{3}, v_{4}, v_{6}, v_{8}\right\}$. From Lemma 2.11, we may assume $N\left(C_{2}\right) \cap N(x)=\left\{v_{0}, v_{1}, v_{4}, v_{7}, v_{8}\right\}$. But now considering $C_{2}$ and the missing edge $v_{0} v_{4}$ of $G[N(x)]$ puts us in the previous case.

(14.4) $G-N[x]$ is 2-connected.

Suppose that $z$ is a cut-vertex of $G-N[x]$. We will show that $z$ must be adjacent to both ends of some missing edge of $G[N(x)]$, contrary to (14.3). Let $C_{1}$ be a component of $G-N[x]-z$, chosen such that $\left|N\left(C_{1}\right) \cap N(x)\right|$ is minimum among all components of $G-N[x]-z$. If $\left|N\left(C_{1}\right) \cap N(x)\right| \geq 7$, then it follows from Lemma 2.12 that for any component $C_{2} \neq C_{1}$ of $G-N[x]-z$, we may contract $C_{1}$ and $C_{2}$ onto $N(x)$ so that $G \geq K_{9}^{=}$, a contradiction. Thus $\left|N\left(C_{1}\right) \cap N(x)\right| \leq 6$. Since $G$ is 6 -connected by (9), we have $\left|N\left(C_{1}\right) \cap N(x)\right| \geq 5$. Then $N\left(C_{1}\right)$ contains both ends of some missing edge of $G[N(x)]$, say $v_{0}, v_{1} \in N\left(C_{1}\right)$. Let $e$ be any missing edge of $G[N(x)]$ such that $v_{0} v_{1}$ and $e$ are disjoint and belong to the same 5-cycle of $\overline{G[N(x)]}$, and suppose neither end of $e$ belongs to $N\left(C_{1}\right)$. We may assume $e=v_{2} v_{3}$. Since $\delta(G) \geq 9$ by (13), $v_{2}$ and $v_{3}$ each have at least two neighbors in $G-N[x]-V\left(C_{1}\right)$. Let $w_{i}$ be a neighbor of $v_{i}$ in $G-N[x]-V\left(C_{1}\right)$ for $i \in\{2,3\}$. We may assume $w_{3} \neq z$. Furthermore, $w_{2} \neq w_{3}$ by (14.3). Since $G-N[x]-V\left(C_{1}\right)$ is connected, there exists a path $P_{1}$ in $G-N[x]-V\left(C_{1}\right)$ with ends $w_{2}, w_{3}$, and a path $P_{2}$ with one end $z$, the other end in $V\left(P_{1}\right)$, and no internal vertices in $V\left(P_{1}\right)$. Possibly, $P_{2}$ consists of only the vertex $z$. Now by contracting $P_{1} \cup P_{2}$ to a single vertex, contracting the edge $v_{2} w_{2}$, and contracting $C_{1}$ onto $v_{1}$, we add the edges $v_{0} v_{1}, v_{1} v_{2}, v_{2} v_{3}$ to $G[N(x)]$, and it follows from Lemma 2.11 that $G \geq K_{9}^{=}$, a contradiction.

Thus at least one end of every missing edge of $G[N(x)]$ disjoint from $v_{0} v_{1}$ and belonging to a 5-cycle of $\overline{G[N(x)]}$ with $v_{0} v_{1}$ must belong to $N\left(C_{1}\right)$. There are eight such missing edges 
of $G[N(x)]$ which give the 8-cycle $v_{2} v_{3} v_{4} v_{9} v_{6} v_{8} v_{5} v_{7}$ of $\overline{G[N(x)]}$. Since $\left|N\left(C_{1}\right) \cap N(x)\right| \leq 6$, either $N\left(C_{1}\right)=\left\{z, v_{0}, v_{1}, v_{2}, v_{4}, v_{5}, v_{6}\right\}$ or $N\left(C_{1}\right)=\left\{z, v_{0}, v_{1}, v_{3}, v_{7}, v_{8}, v_{9}\right\}$. In either case, $N\left(C_{1}\right)$ is a minimal separating set of $G$, so we may put $S=N\left(C_{1}\right)$ and let $G_{1}$ and $G_{2}$ be as defined before (3), where $V\left(G_{1}\right)=V\left(C_{1}\right) \cup N\left(C_{1}\right)$. Let $G_{2}^{\prime}=G_{2}-N[x]$. Suppose $\left\{v_{2}, v_{4}, v_{5}, v_{6}\right\} \subseteq N\left(C_{1}\right)$. Then by contracting $V\left(G_{2}^{\prime}\right) \cup\left\{v_{3}, v_{8}\right\}$ onto $z$, and by contracting the edges $v_{0} x, v_{1} v_{7}$, and $v_{2} v_{9}$, we see $e(G[S])+d_{1}=21$, and by contracting $C_{1}$ onto $v_{0}$, we see $d_{2} \geq 3$. But then by (6), $6 n-20=e(G) \leq 6 n-22$, a contradiction. Thus $\left\{v_{3}, v_{7}, v_{8}, v_{9}\right\} \subseteq N\left(C_{1}\right)$. If $z$ is not adjacent to any vertex of $N\left(C_{1}\right)$, then by contracting $G_{i}-S$ onto $z$, we see $d_{3-i} \geq 6$ for $i \in\{1,2\}$. But then from $(6), e(G) \leq 6 n-22$, again a contradiction. So $z$ has at least one neighbor in $N\left(C_{1}\right) \backslash\{z\}$. By symmetry, we may suppose $z v_{0} \in E(G)$. Now by contracting $V\left(G_{2}^{\prime}\right) \cup\left\{v_{4}, v_{5}\right\}$ onto $z$, and by contracting the edges $x v_{0}$, $v_{2} v_{9}$, and $v_{6} v_{7}$, we see that $e(G[S])+d_{1} \geq 20$. Since $e(G)=6 n-20$, it follows from (6) that $d_{2} \leq 2$, that is $z$ has at least four neighbors in $N\left(C_{1}\right) \backslash\{z\}$. But this requires $z$ to be adjacent to both ends of some missing edge of $G[N(x)]$, contradicting (14.3).

By (2), $v_{0}$ and $v_{2}$ have at least two common neighbors $w_{1}, w_{2} \in V(G) \backslash N[x]$. Similarly, $v_{1}$ and $v_{3}$ have at least two common neighbors $u_{1}, u_{2} \in V(G) \backslash N[x]$. By (14.3), the vertices $w_{1}, w_{2}, u_{1}, u_{2}$ are distinct. By (14.4), there exist two disjoint paths $P_{1}, P_{2}$ with one end in $\left\{w_{1}, w_{2}\right\}$ and the other end in $\left\{u_{1}, u_{2}\right\}$, and all internal vertices in $G-N[x]$. By relabelling if necessary, we may assume $P_{i}$ has ends $w_{i}, u_{i}$ for $i \in\{1,2\}$. Furthermore, there exists a path $Q$ with one end in $V\left(P_{1}\right)$, the other end in $V\left(P_{2}\right)$, and all internal vertices in $G-N[x]$. Now by contracting $P_{1}$ and $P_{2}$ each to a single vertex, contracting $Q$ to a single edge, and contracting the edges $v_{1} u_{1}$ and $v_{2} w_{2}$, we have added the edges $v_{0} v_{1}, v_{1} v_{2}, v_{2} v_{3}$ to $G[N(x)]$, and it follows from Lemma 2.11 that $G \geq K_{9}^{=}$, a contradiction.

(15) $G-N[x]$ is disconnected for any vertex $x \in V(G)$ with $d(x) \in\{9,10,11\}$.

Suppose there exists a vertex $x \in V(G)$ with $d(x) \in\{9,10,11\}$ for which $G-N[x]$ 
is not disconnected. By (14), it follows that $G-N[x]$ must be the empty graph, that is $N[x]=V(G)$. But then $G[N(x)]$ is a graph on at most 11 vertices with $\delta(G[N(x)]) \geq 8$, and so $e(G) \geq 4|G|>5|G|-14$. Thus $G[N(x)] \geq K_{8}^{=}$by Theorem 1.1, a contradiction.

(16) Let $x \in V(G)$ with $d(x) \in\{9,10,11\}$. Then there is no component $C$ of $G-N[x]$ such that $d_{G}(y) \geq 12$ for every $y \in V(C)$.

Suppose such a vertex $x$ and component $C$ exist. Let $G_{1}=G-V(C)$ and $G_{2}=$ $G[V(C) \cup N(C)]$. Let $d_{1}$ be as defined before (5). From (5), $e\left(G_{2}\right) \leq 6\left|G_{2}\right|-20-d_{1}=$ $6(|C|+|N(C)|)-20-d_{1}$. By contracting the edge $x z$, where $z \in N(C)$ has minimum degree $d$ in $G[N(C)]$, we have $d_{1} \geq|N(C)|-d-1$, and hence $e\left(G_{2}\right) \leq 6|C|+5|N(C)|-19+d$. Let $t=e(V(C), N(C))$. Then $e\left(G_{2}\right)=e(C)+t+e(G[N(C)])$. Since $2 e(C) \geq 12|C|-t$ and $2 e(G[N(C)]) \geq d|N(C)|$, we have $2 e\left(G_{2}\right) \geq 12|C|+t+d|N(C)|$. Hence $12|C|+10|N(C)|-$ $38+2 d \geq 2 e\left(G_{2}\right) \geq 12|C|+t+d|N(C)|$, which gives $-t \geq d(|N(C)|-2)-10|N(C)|+38$. Note that $G[N(x)]$ has minimum degree at least 6 by (2), and so $G[N(C)]$ has minimum degree at least $6-\left(d_{G}(x)-|N(C)|\right)$. Thus $d \geq|N(C)|+6-d_{G}(x) \geq|N(C)|-5$. Furthermore, by (9) and (14), $6 \leq|N(C)| \leq d_{G}(x)-1 \leq 10$. It follows that $d(|N(C)|-2)-10|N(C)|>(|N(C)|-$ $5)(|N(C)|-2)-10|N(C)|=|N(C)|^{2}-17|N(C)|+10 \geq-62$. Therefore $-t \geq-24$. Since $2 e(C) \geq 12|C|-t$, we get $e(C) \geq 6|C|-12$. If $|C| \geq 9$, then by the minimality of $G$ we have $C \geq K_{9}^{=}$, a contradiction. Therefore, $|C|<9$. From the inequality $6|C|-12 \leq e(C) \leq\left(\begin{array}{c}|C| \\ 2\end{array}\right)$, it follows that $|C| \leq 2$. Since every vertex of $C$ has degree at least 12 in $G$, and since $d_{G}(x) \leq 11$, it follows that $|C|=2$ and $d_{G}(x)=11$. But now $C$ is a component with $N(C)=N(x)$, contradicting (14).

Now choose a vertex $x \in V(G)$ with $d(x) \in\{9,10,11\}$, such that $G-N[x]$ has a component $C$ of minimum order. Then choose a vertex $y \in V(C)$ of least degree in $G$. By (13) and (16), we have $d_{G}(y) \in\{9,10,11\}$. Let $K$ be the component of $G-N[y]$ containing $x$. We claim that $N(K)$ contains all vertices of $N(y)$ that are not complete to 
all other vertices of $N(y)$. Suppose not, and let $z \in N(y)$ such that $z$ has a nonneighbor in $N(y) \backslash\{z\}$ and $z \notin N(K)$. If $z \in N(x)$, then $z \in N(K)$, and so $z \notin N(x)$, and thus $z \in V(C)$. Therefore $d_{G}(z) \geq d_{G}(y)$ by the choice of $y$. Thus $z$ has some neighbor $z^{\prime} \in N(x) \cup V(C) \backslash N[y]$. Now if $z^{\prime} \notin V(K)$, then the component of $G-N[y]$ containing $z^{\prime}$ would be a proper subgraph of $C$, contradicting our choice of $x$ and $C$. Therefore $z^{\prime} \in V(K)$, and thus $z \in N(K)$, a contradiction. Thus $N(K)$ does contain all vertices of $N(y)$ which are not complete to all other vertices of $N(y)$, but this contradicts (14). This contradiction completes the proof of Theorem 1.2.

\section{Acknowledgment}

Thank you to Zi-Xia Song for introducing me to this topic and for helpful guidance during the early stages of the project.

\section{References}

[1] B. Albar and D. Gonçalves, On triangles in $K_{r}$-minor free graphs, J. Graph Theory 88 (2018) 154-173.

[2] G. A. Dirac, Homomorphism theorems for graphs, Math. Ann. 153 (1964) 69-80.

[3] G. A. Dirac, A property of 4-chromatic graphs and some remarks on critical graphs, J. London Math. Soc. 27 (1952) 85-92.

[4] R. Fabila-Monroy and D. Wood, Rooted $K_{4}$-minors, Elec. J. Combin. 20(2) (2013) \#P64.

[5] E. Györi, On the edge numbers of graphs with Hadwiger number 4 and 5, Period. Math. Hung. 13 (1982) 21-27. 
[6] H. Hadwiger, Über eine Klassifikation der Streckencomplexe, Vierteljschr. Naturforsch. Ges. Zürich. 88 (1943) 133-142.

[7] I. T. Jakobsen, A homomorphism theorem with an application to the conjecture of Hadwiger, Studia Sci. Math. Hungar. 6 (1971) 151-160.

[8] I. T. Jakobsen, On certain homomorphism properties of graphs I, Math. Scand. 31 (1972) 379-404.

[9] I. T. Jakobsen, On certain homomorphism properties of graphs II, Math. Scand. 52 (1983) 229-261.

[10] L. K. Jørgensen, Contractions to $K_{8}$, J. Graph Theory. 18 (1994) 431-448.

[11] W. Mader, Homomorphiesätze für Graphen, Math. Ann. 178 (1968) 154-168.

[12] N. Robertson, P. Seymour, and R. Thomas, Hadwiger's conjecture for $K_{6}$-free graphs, Combinatorica. 13 (1993) 279-361.

[13] M. Rolek, Graphs with no $K_{9}^{=}$minor are 10-colorable, in preparation.

[14] M. Rolek, Computer search details: Extremal function for $K_{9}^{=}$minors, https://msrolek.sites.wm.edu/wp-content/uploads/sites/317/2018/04/K9Minus2ComputerSear

[15] M. Rolek and Z-X. Song, Coloring graphs with forbidden minors, J. Combin. Theory, Ser. B. 127 (2017) 14-31.

[16] P. Seymour, Disjoint paths in graphs, Disc. Math. 29 (1980) 293-309.

[17] Z-X. Song, The extremal function for $K_{8}^{-}$minors, J. Combin. Theory, Ser. B. 95 (2005) $300-317$.

[18] Z-X. Song, Extremal functions for contractions of graphs, Doctoral Dissertaton, Georgia Institute of Technology, (2004). 
[19] Z-X. Song and R. Thomas, The extremal function for $K_{9}$ minors, J. Combin. Theory, Ser. B. 96 (2006) 240-252.

[20] R. Thomas and Y. Yoo, The extremal functions for triangle-free graphs with excluded minors, arXiv:1801.06887.

[21] C. Thomassen, 2-linked graphs, Eur. J. Combin. 1 (1980) 371-378.

[22] P. Turán, On an extremal problem in graph theory, Matematikai és Fizikai Lapok. 48 (1941) 436-452.

[23] K. Wagner, Über eine Eigenschaft der ebenen Komplexe, Math. Ann. 114 (1937) 570590.

[24] D. R. Woodall, A Short Proof of a Theorem of Dirac's About Hadwiger's Conjecture, J. Graph Theory. 16 (1992) 79-80. 